\title{
Comparação experimental dos algoritmos coletivos para o MPI_Allgather no OpenMPI
}

\author{
Wilton Jaciel Loch ${ }^{1}$, Guilherme Piêgas Koslovski ${ }^{1}$ \\ ${ }^{1}$ Programa de Pós-Graduação em Computação Aplicada (PPGCA) \\ Universidade do Estado de Santa Catarina (UDESC) - Joinville, SC - Brasil \\ Resumo. Uma das chamadas coletivas populares trata-se da MPI_Allgather, \\ cuja implementação seleciona um algoritmo dentre os vários disponíveis com \\ base nos parâmetros de execução e utilizando regras estáticas. $O$ objetivo deste \\ trabalho é avaliar experimentalmente as regras de seleção de algoritmo no \\ OpenMPI 4.0.3 para esta chamada. Os resultados obtidos apontam que as es- \\ colhas são ineficientes para os cenários avaliados em grande parte dos testes.
}

\section{Introdução e metodologia experimental}

As comunicações coletivas no Message Passing Interface (MPI) representam uma das principais formas de trocas de mensagem e dividem-se entre envios de um para muitos e muitos para muitos [MPI Forum 2015]. Para ambas as formas há uma multitude de algoritmos com desempenhos variáveis, cuja escolha depende dos parâmetros de execução. Experimentos foram realizados comparando os algoritmos disponíveis para a chamada coletiva MPI_Allgather na implementação OpenMPI 4.0.3, que no momento da escrita deste trabalho trata-se da versão de distribuição mais recente. Os algoritmos utilizados nesta chamada são: linear, bruck, recursive doubling, ring, neighbor exchange e two proc. O algoritmo two proc tem sua operação limitada apenas a dois processos, e portanto foi removido dos testes. De forma semelhante, o algoritmo recursive doubling opera apenas com quantidades de processos que são potências de dois, e portanto foi mantido apenas nos testes pertinentes. São utilizadas 62 e 128 como quantidades de processos (distribuídos ciclicamente) para representar casos gerais de valores pares e potências de dois, respectivamente. A quantidade de dados enviados é variada entre 32 Bytes e 512 KiloBytes para englobar blocos pequenos, médio e grandes, em multiplicações sucessivas por 4 devido ao limite de espaço. O tempo de execução é medido através de contadores próprios do MPI (MP I_Wtime) em aplicações ad hoc. Cada configuração de teste é repetida 50 vezes. Os experimentos foram realizados em 2 computadores homogêneos do aglomerado Cervino da Universidade de Neuchâtel (Unine). Ambos possuem 2 processadores Intel Xeon E5-2683 v4 (16 núcleos cada) e 128GB de memória. Ambas as máquinas são conectadas por uma rede Ethernet de 40Gbps.

\section{Resultados}

A Tabela 1 apresenta os resultados dos experimentos e é dividida em duas seções relativas às quantidades de processos. $\mathrm{O}$ tempo de cada algoritmo nas diferentes cargas é mostrado em dezenas de milissegundos e as células dos menores e maiores resultados de cada carga são coloridas em verde e vermelho, respectivamente. A linha intitulada OpenMPI representa as escolhas de algoritmo feitas pela implementação e os escolhidos para cada carga são destacados em negrito. Por fim, na mesma linha e em cada célula, uma flecha ascendente representa a escolha do melhor algoritmo enquanto uma descendente a escolha do pior. 


\begin{tabular}{|c|c|c|c|c|c|c|c|c|c|}
\hline & \multicolumn{9}{|c|}{ Tempo MP I_Al lgather $(\times 10 \mu \mathrm{s})$} \\
\hline & Algoritmo & $32 \mathrm{~B}$ & $128 \mathrm{~B}$ & $512 \mathrm{~B}$ & $2 \mathrm{~KB}$ & $8 \mathrm{~KB}$ & $32 \mathrm{~KB}$ & $128 \mathrm{~KB}$ & $512 \mathrm{~KB}$ \\
\hline \multirow{5}{*}{ 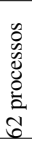 } & OpenMPI & $32 \pm 153$ & $31 \pm 152$ & $\uparrow 36 \pm 154$ & $246 \pm 177$ & $359 \pm 174$ & $1583 \pm 176$ & $6467 \pm 4477$ & $25573 \pm 4736$ \\
\hline & Linear & $49 \pm 288$ & $67 \pm 295$ & $94 \pm 293$ & $206 \pm 285$ & $1729 \pm 5040$ & $2801 \pm 4797$ & $7728 \pm 4053$ & $23723 \pm 4873$ \\
\hline & Bruck $(0-2)$ & $29 \pm 149$ & $32 \pm 153$ & $45 \pm 153$ & $53 \pm 153$ & $100 \pm 158$ & $451 \pm 149$ & $3067 \pm 216$ & $12461 \pm 1366$ \\
\hline & Ring & $306 \pm 151$ & $266 \pm 176$ & $242 \pm 152$ & $304 \pm 166$ & $451 \pm 197$ & $1388 \pm 446$ & $5738 \pm 2920$ & $29007 \pm 8456$ \\
\hline & Neighbor exchange (3-7) & $129 \pm 157$ & $118 \pm 165$ & $134 \pm 162$ & $\mathbf{2 4 2} \pm \mathbf{1 6 1}$ & $392 \pm 264$ & $1675 \pm 305$ & $6116 \pm 3775$ & $26317 \pm 6144$ \\
\hline \multirow{6}{*}{$\begin{array}{l}0 \\
0 \\
0 \\
0 \\
0 \\
0 \\
0 \\
0 \\
0 \\
\mathbb{1}\end{array}$} & OpenMPI & $\uparrow 67 \pm 418$ & $45 \pm 208$ & $799 \pm 775$ & $1052 \pm 421$ & $1831 \pm 742$ & $5994 \pm 251$ & $27294 \pm 6882$ & $104481 \pm 16336$ \\
\hline & Linear & $92 \pm 595$ & $208 \pm 721$ & $166 \pm 428$ & $656 \pm 744$ & $4590 \pm 10770$ & $9665 \pm 9677$ & $35678 \pm 16620$ & $292064 \pm 7955$ \\
\hline & Bruck & $209 \pm 578$ & $52 \pm 201$ & $93 \pm 436$ & $211 \pm 548$ & $716 \pm 514$ & $3462 \pm 548$ & $14270 \pm 1270$ & $52994 \pm 6152$ \\
\hline & Recursive doubling (0-2) & $\mathbf{5 2} \pm \mathbf{3 2 3}$ & $122 \pm 456$ & $104 \pm 574$ & $74 \pm 211$ & $265 \pm 301$ & $1450 \pm 403$ & $5798 \pm 401$ & $25609 \pm 3360$ \\
\hline & Ring & $669 \pm 233$ & $793 \pm 578$ & $684 \pm 595$ & $976 \pm 714$ & $1610 \pm 622$ & $6626 \pm 3970$ & $32383 \pm 11268$ & $147311 \pm 25497$ \\
\hline & Neighbor exchange (3-7) & $347 \pm 701$ & $379 \pm 736$ & $507 \pm 707$ & $574 \pm 719$ & $3952 \pm 6618$ & $\mathbf{7 1 9 0} \pm \mathbf{5 3 7 9}$ & $27648 \pm 7682$ & $101899 \pm 12278$ \\
\hline
\end{tabular}

Tabela 1. Tempos de execução da chamada MPI Allgather.

Inicialmente, é válido destacar que o melhor desempenho alterna entre o bruck e o recursive doubling em todas as cargas, enquanto o pior desempenho alterna entre o ring e linear. Este comportamento é explicado pelos custos teóricos dos algoritmos, ambos ring e neighbor exchange tem complexidades lineares, bruck e recursive doubling possuem complexidades logarítmicas e o linear complexidade quadrática, justificando suas ascenções vertiginosas de tempo com o crescimento dos dados. Os algoritmos de complexidade linear são empregados pelo OpenMPI porque embora o recursive doubling e bruck possuam complexidades teóricas logarítmicas, na prática seus padrões de comunicação são difusos e para o último são ainda necessárias cópias e movimentações de memória em sua operação. Estes fatores tornam seus tempos de execução maiores que os competidores com o crescimento da infraestrutura, processos e dados. Portanto, a realização dos testes com grandezas maiores para as variáveis e mais máquinas interligadas tenderia a uma superação dos algoritmos lineares sobre o bruck e o recursive doubling. Por fim, é possível verificar que as escolhas determinísticas do OpenMPI são ineficientes para o ambiente de testes empregado. Com 62 processos, em apenas 3 das 8 cargas o algoritmo escolhido era de fato o melhor, ao passo que um algoritmo sub-ótimo foi escolhido nos casos restantes, resultando em escolhas ineficientes em 5 dos 8 testes. Com 128 processos os resultados são ainda piores, já que em apenas um dos testes o melhor algoritmo foi escolhido e nos restantes foram tomados algoritmos sub-ótimos, implicando em escolhas ineficientes em 7 dos 8 casos.

\section{Considerações Finais}

Os resultados dos testes demonstram que há de fato ineficiência na seleção de algoritmos para a operação coletiva MP I_Al lgather e sugerem que problemas semelhantes podem também existir para outras chamadas. De forma geral, este trabalho serve de justificativa e ponto de partida para o desenvolvimento de ferramentas de auto ajuste dos parâmetros para seleção de algoritmos. As observações preconizam o desenvolvimento de novos algoritmos que tenham complexidade logarítmica, comunicação local e não façam cópias e movimentações de memória, unindo as vantagens de diferentes algoritmos já existentes. Como trabalhos futuros se indica a execução de testes maiores e o desenvolvimento de algoritmos mais eficientes e ferramentas inteligentes para a escolha.

Agradecimentos: Este trabalho foi desenvolvido com financiamento da FAPESC e apoio da Unine através do acesso à infraestrutura de testes.

\section{Referências}

MPI Forum (2015). MPI: A Message-Passing Interface Standard. 